\title{
AVALIAÇÃO POR TCFC DO TRANSPORTE DO CANAL RADICULAR APÓS O USO DE DIFERENTES SISTEMAS ROTATÓRIOS DE NITI
}

\author{
CBCT ASSESSMENT OF THE ROOT CANAL TRANSPORTATION AFTER \\ THE USE OF DIFFERENT NITI ROTARY SYSTEMS
}

\author{
Gustavo Silva CHAVES'; Vinicius Caixeta de SOUSA'; Marco Antônio Zaiden LOUREIRO'; Giulliano Caixeta SERPA'; \\ Maria Luiza Lima SANTANA'; Orlando Aguirre GUEDES ${ }^{2}$ \\ 1 - Faculdade de Odontologia, Universidade Federal de Goiás. \\ 2 - Faculdade de Odontologia, Centro Universitário de Anápolis-UniEVANGÉLICA
}

\section{RESUMO}

Objetivo: o objetivo do estudo foi avaliar a ocorrência de transporte do canal radicular após o preparo com instrumentos de níquel-titânio (NiTi) de rotação contínua, por meio do exame de tomografia computadorizada de feixe cônico (TCFC). Material e método: Canais mesiovestibulares e mesiolinguais de vinte molares inferiores humanos foram distribuídos aleatoriamente em dois grupos experimentais $(n=10)$, de acordo com o sistema rotatório de NiTi: Grupo 1 - BioRace; e Grupo 2 - ProTaper Next. Imagens de TCFC foram obtidas antes do preparo do canal radicular (TCFC 1) e após o uso dos instrumentos BR2 (\#25/.04) e X2 (\#25/.06) (TCFC 2) e BR5 (\#40/.04) e X4 (\#40/.06) (TCFC 3). Dois examinadores avaliaram todas as imagens determinando a ocorrência ou não de transporte do canal radicular. Foram estabelecidos 3 níveis para a mensuração nas imagens: 1-2 mm aquém do vértice radicular (terço apical); 3-4 $\mathrm{mm}$ aquém do vértice radicular (terço médio); 4- $2 \mathrm{~mm}$ abaixo da furca (terço cervical). Os dados foram analisados utilizando o teste de Kruskal-Wallis com pós-teste de Dunn para comparações múltiplas. O nível de significância foi de $5 \%$. Resultados: Os canais radiculares preparados com o sistema BioRace não apresentaram transporte $(\mathrm{P}<0,05)$. Foi observado transporte apenas no terço apical do canal mesiovestibular após o uso do instrumento ProTaper Next X4 (\#40/.06) $(\mathrm{P}<0,05)$. Conclusão: $\mathrm{O}$ sistema BioRace permitiu a ampliação dos canais mesiovestibular e mesiolingual de molares inferiores até o diâmetro cirúrgico 40 sem resultar em transporte do canal.

Palavras-chave: Endodontia, Instrumentos Odontológicos, Tomografia Computadorizada de Feixe Cônico.

\section{INTRODUÇÃO}

O preparo do canal radicular consiste em uma das etapas mais importantes da terapia endodôntica. Esta fase do tratamento é responsável por conferir ao conduto um formato cônico e afunilado em sentido apical, o que auxilia na sanificação e obturação ${ }^{1}$. No entanto, a instrumentação de dentes apresentando curvaturas acentuadas representa um verdadeiro desafio ao clínico, visto a possibilidade da ocorrência de erros operatórios, como por exemplo, o desvio do conduto do seu eixo original ${ }^{2-4}$. A habilidade de um instrumento ou técnica em permanecer centralizado na trajetória original do canal é uma característica positiva e amplamente valorizada nos dias atuais ${ }^{3,5}$.

A introdução dos instrumentos rotatórios de níquel-titânio (Ni-Ti) resultou no aprimoramento da modelagem dos canais radiculares, bem como, na redução de iatrogênias. ${ }^{4}$ Além disso, esses instrumentos apresentam maior capacidade em permanecer centralizados no canal radicular, o que resulta em preparos mais homogêneos ${ }^{6}$. Essas características têm sido associadas à obtenção de resultados satisfatórios na clínica endodôntica. ${ }^{7}$

Vários métodos têm sido propostos na avaliação da capacidade dos instrumentos de NiTi em manter a forma original do canal e a sua tendência em produzir o transporte do conduto, como por exemplo: histológico, radiográfico, anatômico seccional e microscopia eletrônica de varredura ${ }^{8,9}$. Entretanto, a impossibilidade da realização de análises simultâneas dos diferentes parâmetros relacionados ao preparo do canal radicular e a perda de amostras, impõem limites ao emprego destes métodos ${ }^{8,9}$.

A tomografia computadorizada de feixe cônico (TCFC) tem sido utilizada em várias áreas da Endodontia ${ }^{10}$. A possibilidade da redução ou eliminação da sobreposição de estruturas anatômicas torna a TCFC superior à radiografia periapical convencional $^{11}$. Em comparação à tomografia médica, a TCFC apresenta algumas vantagens: menor dose de radiação, imagem de alta resolução e maior precisão na mensuração do volume nos diferentes planos em virtude do voxel ser isotrópico ${ }^{12}$.

Possíveis erros operatórios, que podem afetar o prognóstico da terapia endodôntica, devem ser considerados e avaliados antes da escolha e utilização de novos instrumentos ${ }^{13}$. Assim, o objetivo do presente estudo foi avaliar a ocorrência de transporte do canal radicular após o preparo com instrumentos de NiTi de rotação contínua, por meio da TCFC. As hipóteses nulas testadas foram de que não haveria diferença significativa na ocorrência de transporte do canal em função (i) do sistema rotatório de NiTi, (ii) do canal radicular, e (iii) terço da raiz. 


\section{MATERIAL E MÉTODO}

O protocolo do estudo foi devidamente registrado na Comissão Nacional de Ética em Pesquisa (CONEP) e aprovado pelo Comitê de Ética em Pesquisa da Universidade Federal de Goiás (CAAE 73430617.9.0000.5083).

\section{Seleção das amostras}

Molares humanos inferiores, extraídos por diferentes razões, foram obtidos no Serviço de Urgência da Faculdade de Odontologia da Universidade Federal de Goiás, Goiânia, GO, Brasil. Os dentes foram armazenados em solução de timol à $0,2 \%$, sendo em seguida imersos, por 30 minutos, em solução de hipoclorito de sódio à 5\% (NaOCl; ASFER Indústria Química Ltda., São Caetano do Sul, SP, Brasil), para remoção de tecidos orgânicos da superfície externa das raízes.

Tomadas radiográficas pré-operatórias foram realizadas no intuito de confirmar a ausência de calcificações no interior dos canais radiculares, tratamento endodôntico prévio, retentor intrarradicular e reabsorções internas e externas, bem como a presença da completa formação dos ápices dentários. As radiografias periapicais foram obtidas pela técnica do paralelismo, utilizando o aparelho Spectro X70 (Dabi Atlante, Ribeirão Preto, $\mathrm{SP}$, Brasil), com tubo focal de 0,8 $\mathrm{mm} \times 0,8 \mathrm{~mm}$ e filmes Kodak Insight-E (Eastman Kodak Co., Rochester, NY, EUA). Em todos os dentes foi utilizada uma plataforma radiográfica para a padronização das imagens radiográficas. Os filmes foram processados em uma processadora automática e a avaliação das imagens foi realizada em negatoscópio sob penumbra com auxílio de lupa.

Imagens de TCFC pré-operatórias (TCFC 1) foram obtidas pelo sistema Eagle 3D (Dabi Atlante, Ribeirão Preto, SP, Brasil). As imagens foram capturadas com FOV de $80 \times 120 \mathrm{~mm}$, voxel de 0,2 mm, 33,5 segundos de exposição com 1024 visualizações, tensão de tubo de $90 \mathrm{kVp}$ e corrente de $5 \mathrm{~mA}$, e examinadas com auxílio do software OsiriX ${ }^{\circledR}$ DICOM Viewer versão 8.0.2 (Pixmeo SARL, Bernex, Suíça) em um computador MacBook Air (Apple Inc., Santa Clara, CA, EUA), com sistema operacional MacOS Sierra versão 10.12.1 (Apple Inc., Santa Clara, CA, EUA), com processador Intel Core $i 5^{\circledR} 1,4 \mathrm{Ghz}$ (Intel Corporation, Santa Clara, CA, EUA), placa de vídeo Intel HD Graphics 5000 (Intel Corporation, Santa Clara, CA, EUA) e monitor de 13,3 polegadas resolução 1440 × 900 .

As raízes foram posicionadas de forma a manter todos os ápices radiculares em um mesmo nível e garantir o reposicionamento dos espécimes durante novas aquisições de TCFC. Foram utilizados neste estudo apenas dentes apresentando 3 canais radiculares ( 1 mesiovestibular, 1 mesiolingual e 1 distal). Todos os dentes apresentavam comprimento igual ou inferior a $22 \mathrm{~mm}$ e raiz mesial com raio de curvatura maior que $4 \mathrm{~mm}$ e menor que $8 \mathrm{~mm}^{14}$. Após aplicação dos critérios de inclusão, 20 dentes foram selecionados.

Abertura coronária, determinação do comprimento de trabalho, divisão dos grupos experimentais e preparo dos canais radiculares

Após a obtenção das imagens de TCFC 1, realizou-se o procedimento de abertura coronária com broca diamantada esférica \#1013 HL (KG Sorensen, Barueri, SP, Brasil). A forma de conveniência foi realizada com broca Endo Z (Dentsply/Maillefer, Ballaigues, Suíça). Ambas as brocas foram utilizadas sob refrigeração e em alta rotação (Kavo Ind. Com. Ltda., Joinville,
SC, Brasil). A exploração e o esvaziamento dos canais radiculares foram realizados com limas do tipo K \#10 e \#15 (Dentsply/ Maillefer, Ballaigues, Suíça). Dentes apresentando diâmetro anatômico superior a $0,15 \mathrm{~mm}$ foram substituídos. O comprimento de trabalho (CT) foi determinado com o uso de limas K-flexofile (\#10 e \#15) (Dentsply Maillefer, Ballaigues, Suíça) introduzidas até serem observadas no forame apical. O CT foi estabelecido a $1 \mathrm{~mm}$ aquém do forame apical. $\mathrm{O}$ alargamento dos terços cervical e médio foi realizado com instrumento Pre Race (30/.06) (FKG Dentaire, La Chaux-de-Fonds, Suíça) acionado por motor elétrico (X-Smart Plus ${ }^{\circledR}$; Dentsply/Maillefer, Ballaigues, Suíça), e de acordo com os padrões pré-determinados pelo fabricante. Em seguida, os vinte dentes foram aleatoriamente distribuídos em dois grupos experimentais, de 10 dentes cada, de acordo com o sistema de NiTi a ser utilizado para o preparado: G1 - BioRaCe (FKG Dentaire, La Chaux-de-Fonds, Suíça); e G2 - ProTaper Next (Dentsply/Maillefer, Ballaigues, Suíça).

Para o preparo dos canais mesiovestibular e mesiolingual, os dentes foram adaptados e estabilizados individualmente em uma morsa (Metalsul, Joinville, SC, Brasil), a fim de permitir a execução mais próxima do ideal de cada técnica. No grupo 1 foram utilizados os instrumentos BR1 (\#15/.05), BR2 (\#25/.04), BR3 (\#25/.06), BR4 (\#35/.04) e BR5 (\#40/.04). No grupo 2 a sequência de instrumentos utilizada foi X1 (\#17/.04), X2 (\#25/.06), X3 (\#30/.07) e X4 (\#40/.06). Os instrumentos foram acoplados a um motor elétrico (X-Smart Plus ${ }^{\circledR}$; Dentsply/Maillefer, Ballaigues, Suíça) e acionados seguindo os padrões determinados pelo fabricante. Cada instrumento foi utilizado para preparar cinco dentes. Uma vez que o instrumento alcançou o comprimento de trabalho em rotação livre, este foi removido. Neste ponto, o instrumento seguinte da sequência foi utilizado. Esta manobra foi repetida até a finalização do preparo.

Cada dente foi submetido a dois novos escaneamentos. Um logo após o preparo com os instrumentos BR2 (\#25/.04) e X2 (\#25/.06) (TCFC 2) e outro após o preparo com os instrumentos BR5 (\#40/.04) e X4 (\#40/.06) (TCFC 3). As TCFCs 2 e 3 foram adquiridas seguindo os mesmos parâmetros da TCFC 1 .

Durante a instrumentação e a cada troca de lima, os canais foram irrigados com $3 \mathrm{~mL}$ de $\mathrm{NaOCl}$ à 2,5\% (ASFER Indústria Química Ltda., São Caetano do Sul, SP, Brasil) com o auxílio de seringa plástica de $5 \mathrm{~mL}$ (Ultradent Products Inc., South Jordan, UT, EUA) e agulha para irrigação EndoEze ${ }^{\circledR}$ (Ultradent Products Inc., South Jordan, UT, EUA). Os canais foram secados com pontas de papel absorventes esterilizadas (Dentsply/Maillefer, Ballaigues, Suíça) e preenchidos com $5 \mathrm{~mL}$ de EDTA à 17\% (pH 7,2) (Biodinâmica, Ibiporã, PR, Brasil) por 3 minutos, para remoção da smear layer. Ao final, uma nova irrigação com $3 \mathrm{~mL}$ de $\mathrm{NaOCl}$ à 2,5\% (ASFER Indústria Química Ltda., São Caetano do Sul, SP, Brasil) foi realizada. Um especialista em Endodontia com mais de 5 anos de experiência realizou todas as etapas clínicas.

\section{Análise das imagens de TCFC}

A análise das imagens foi realizada utilizando metodologia modificada daquela proposta por Mamede-Neto et al.15 (2017). O índice de transporte do canal radicular foi determinado no sentido mesiodistal e correspondeu à variação, em milímetros, do desvio do eixo central do canal radicular após a instrumentação. O transporte do canal radicular foi estabelecido a partir da 
mensuração da menor distância entre as paredes mesial e distal do canal radicular e a superfície externa radicular mesial e distal, antes (TCFC1 - M1 e D1) e após os preparos do canal radicular (TCFC2 - M2 e D2) (TCFC3 - M3 e D3).

Os níveis estabelecidos para a realização das mensurações nas imagens dos canais radiculares foram: 1-2 $\mathrm{mm}$ aquém do vértice radicular (terço apical); 3 - $4 \mathrm{~mm}$ aquém do vértice radicular (terço médio); 4- 2 mm abaixo da furca (terço cervical). Para a realização das mensurações foi empregado o software OsiriX ${ }^{\circledR}$ DICOM Viewer versão 8.0.2 (Pixmeo SARL, Bernex, Suíça) em um computador MacBook Air (Apple Inc., Santa Clara, CA, EUA), com sistema operacional MacOS Sierra versão 10.12.1 (Apple Inc., Santa Clara, CA, EUA), com processador Intel Core $15^{\circledR} 1,4$ Ghz (Intel Corporation, Santa Clara, CA, EUA), placa de vídeo Intel HD Graphics 5000 (Intel Corporation, Santa Clara, CA, EUA) e monitor de 13,3 polegadas resolução 1440 x 900. Para facilitar as mensurações, ajustes de ampliação, brilho e contraste disponíveis no programa foram utilizados. Dois examinadores, um radiologista e um endodontista, foram calibrados em $10 \%$ da amostra. A mensuração das imagens foi realizada duas vezes, com intervalo de duas semanas entre a primeira e a segunda avaliação.

\section{Análise estatística}

Os dados coletados foram tabulados para realização da análise estatística dos resultados. As análises estatísticas foram realizadas com o auxílio do programa IBM SPSS for Windows 21.0 (IBM Corporation, Somers, NY, EUA). Devido à distribuição não-paramétrica dos dados (teste de Shapiro-Wilk) e não homogênea (teste de Levene), foi realizado o teste de Kruskal-Wallis com pós-teste de Dunn para comparações múltiplas. O nível de significância estabelecido foi de $p<0,05$. A concordância para as alterações identificadas pelos dois examinadores foi avaliada pela análise de concordância Kappa.

\section{RESULTADOS}

O valor Kappa foi de 0,84, o que indicou excelente grau de concordância interexaminador. A frequência e análise estatística da ocorrência de transporte do canal radicular considerando os instrumentos rotatórios de Ni-Ti utilizados no preparo, canal e terço da raiz está descrita na Tabela 1 . Os canais radiculares instrumentados com o sistema BioRaCe não apresentaram transporte $(\mathrm{P}<0,05)$. No terço apical do canal mesiovestibular foi observado transporte do canal $(30,0 \%)$ após o uso do instrumento ProTaper Next X4 (\#40/.06) $(\mathrm{P}>0,05)$.

Tabela 1 - Frequência da ocorrência de transporte do canal radicular em função do tipo de sistema rotatório de $\mathrm{NiTi}$, canal radicular e terço da raiz utilizando a tomografia computadorizada de feixe cônico.

\begin{tabular}{|c|c|c|c|c|c|}
\hline \multirow{2}{*}{ Sistema/Instrumento } & \multirow{2}{*}{ Canal } & \multicolumn{3}{|l|}{ Terço } & \multirow{2}{*}{ Valor de P } \\
\hline & & Cervical & Médio & Apical & \\
\hline \multirow{2}{*}{ BioRace/BR2 } & MV & $0,0 \%$ & $0,0 \%$ & $0,0 \%$ & \multirow{2}{*}{$P>0,05$} \\
\hline & ML & $0,0 \%$ & $0,0 \%$ & $0,0 \%$ & \\
\hline \multirow{2}{*}{ BioRace/BR5 } & MV & $0,0 \%$ & $0,0 \%$ & $0,0 \%$ & \multirow{2}{*}{$P>0,05$} \\
\hline & $M L$ & $0,0 \%$ & $0,0 \%$ & $0,0 \%$ & \\
\hline \multirow{2}{*}{ ProTaper Next/X2 } & MV & $0,0 \%$ & $0,0 \%$ & $0,0 \%$ & \multirow{2}{*}{$P>0,05$} \\
\hline & $M L$ & $0,0 \%$ & $0,0 \%$ & $0,0 \%$ & \\
\hline \multirow{2}{*}{ ProTaper Next/X4 } & MV & $0,0 \%$ & $0,0 \%$ & $30,0 \%$ & \multirow{2}{*}{$P<0,05$} \\
\hline & $M L$ & $0,0 \%$ & $0,0 \%$ & $0,0 \%$ & \\
\hline Valor de P & & $P>0,05$ & $P>0,05$ & $P<0,05$ & \\
\hline
\end{tabular}

NiTi- Níquel-titânio; MV- mesiovestibular; ML- mesiolingual; D- distal.

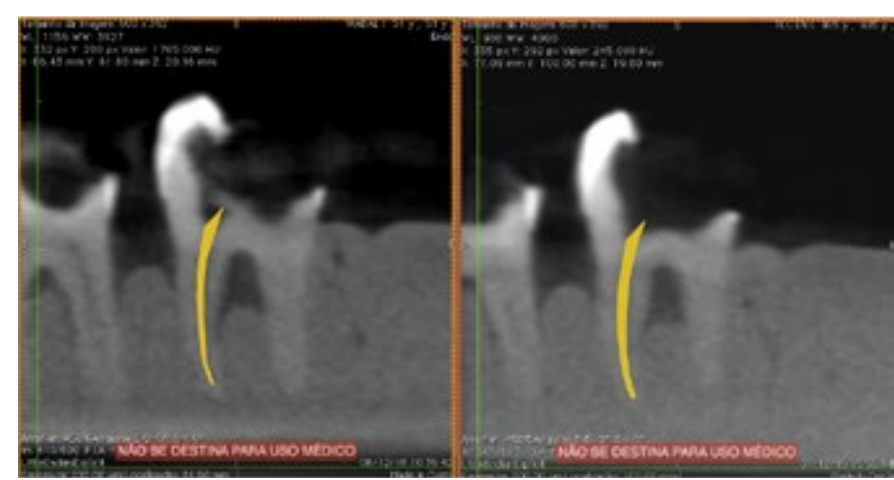

Figura 1 - Imagens de tomografia inicial (à esquerda) e após o preparo com instrumento de ponta \#25 (à direita) do grupo 1, mostrando manutenção de forma. A forma do canal radicular foi destacada em amarelo.

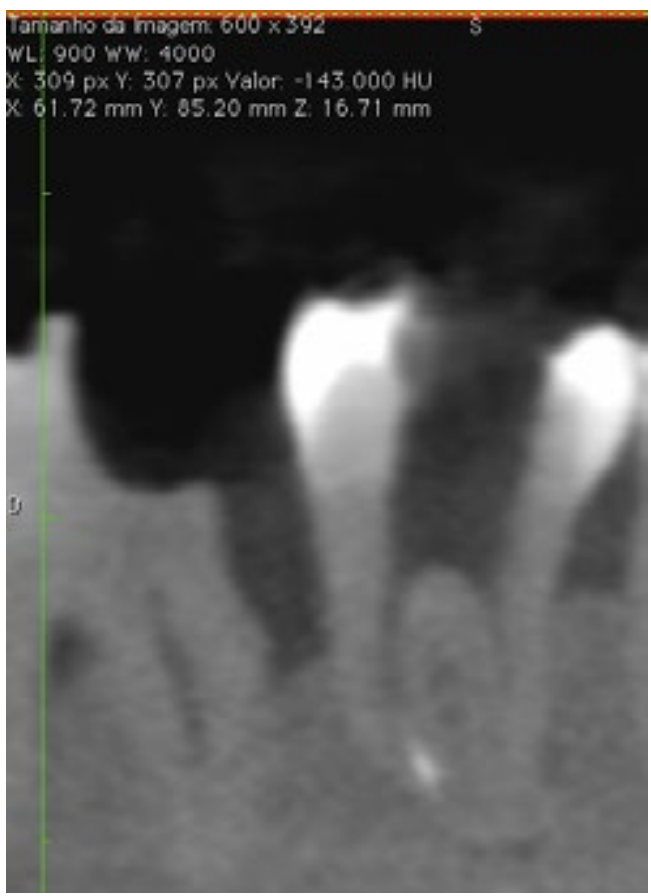

Figura 2 - Imagem de tomografia evidenciando um instrumento fraturado durante o preparo do canal.

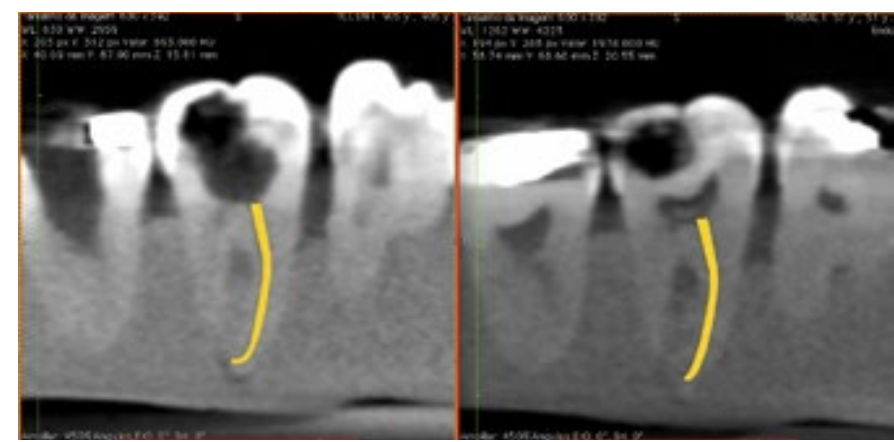

Figura 3 - Imagens de tomografia após o preparo com instrumento \#25 (à esquerda) e após o preparo com instrumento de ponta $\# 40$ (à direita) do grupo 2, mostrando alteração de forma no terço apical. A forma do canal radicular foi destacada em amarelo. 


\section{DISCUSSÃO}

O preparo do canal radicular é uma das etapas mais importantes da terapia endodôntica. Uma modelagem correta contribui para uma adequada ação dos irrigantes e medicação intracanal, bem como, na realização de uma obturação satisfatória do sistema de canais radiculares ${ }^{4,16}$. O objetivo do presente estudo foi avaliar a ocorrência de transporte do canal radicular após o preparo com dois sistemas de NiTi de rotação contínua. Os resultados demonstraram que o tipo de instrumento utilizado no preparo e canal radicular e terço da raiz preparados influenciaram na ocorrência de transporte do canal. Assim, todas as hipóteses nulas testadas foram rejeitadas.

No presente estudo, imagens de TCFC foram utilizadas para avaliar a modelagem e ocorrência de transporte do canal nos grupos testados. A TCFC é um método não-invasivo, relevante para a análise da anatomia do canal e para avaliar a técnica de preparo. Por meio dessa ferramenta, é possível analisar a morfologia tridimensional do canal antes e após o preparo ${ }^{10,17}$. Estudos prévios utilizaram canais simulados ou dentes extraídos como modelos experimentais ${ }^{22,23}$. O uso de dentes humanos extraídos apresenta vantagens sobre os canais simulados em blocos de resina por apresentarem condições mais semelhantes às situações clínicas $^{22}$. Apesar disso, o uso de canais simulados neste tipo de trabalho permite maior padronização do comprimento do canal, do diâmetro e do raio de curvatura ${ }^{23}$.

Devido às propriedades elásticas da liga, os instrumentos rotatórios de NiTi facilitaram e simplificaram os procedimentos de preparo do canal. Uma vasta gama de instrumentos tem sido desenvolvida, fornecendo ao clínico várias opções para um satisfatório preparo e modelagem do canal radicular. Esses sistemas diferem entre si pela seção transversal, ângulo de corte, ângulo helicoidal, guia radial, sequência de instrumentação e dinâmica de movimento ${ }^{24}$. No presente trabalho, foram utilizados dois sistemas automatizados de instrumentação, ambos de rotação contínua: os sistemas BioRace e Protaper Next. O transporte do canal na região apical foi observado somente no grupo 2 , após o preparo com o instrumento X4 (\#40/.06). Este instrumento apresenta conicidade media de $60 \%$, o que confere maior resistência à fratura, porém menor flexibilidade. Estudos prévios demonstraram que instrumentos com maior conicidade devem ser implementados com outros de menor conicidade e portanto mais flexíveis, para evitar o transporte e desvio em canais com curvaturas acentuadas ${ }^{17,18,19}$. De acordo com Buchanan $(2001)^{21}, \mathrm{o}$ transporte de canal ocorre mais acentuadamente em dilatações da região apical maiores do que \#25, devido à maior quantidade de núcleo metálico desses instrumentos. Portanto, parece ser mais adequado que preparos apicais acima desse calibre sejam realizados com instrumentos de pouca conicidade, assegurando assim uma dilatação apropriada ao diâmetro apical anatômico de cada grupo dentário, mantendo a forma original do canal, satisfazendo assim o objetivo mecânico proposto por Schilder para o preparo do canal e ao mesmo tempo a remoção satisfatória de dentina ${ }^{1}$.

No presente trabalho, houve fratura de 3 instrumentos BR3 (\#25/.06), e um instrumento X4 (\#40/.06). Mesmo esta análise não sendo objetivo desse estudo, esta intercorrência pode ter ocorrido devido às repetidas utilizações de um mesmo instrumento para o preparo dos canais. Embora, até o momento, não exista um consenso a respeito do número de usos dos instrumentos de
$\mathrm{Ni}$-Ti, o uso de um mesmo kit no preparo de até 5 molares extraídos pode ser apontado como uma desvantagem deste estudo, já que esses dentes podem se apresentar mais desidratados e resistentes ao corte ${ }^{18,19,20}$. Outra limitação pode estar relacionada ao tamanho reduzido da amostra. Assim, novos estudos devem ser realizados com vistas ao desenvolvimento de novos instrumentos/técnicas que possibilitem a realização de preparos do canal radicular com baixos índices de iatrogenias.

\section{CONCLUSÃO}

O sistema BioRace permitiu a ampliação dos canais mesiovestibular e mesiolingual de molares inferiores até o diâmetro cirúrgico 40 sem resultar em transporte do canal radicular.

\section{REFERÊNCIAS}

01. Schilder H. Cleaning and shaping the root canal. Dent Clin North Am.1974; 18:269-96.

02. Abou-Rass M, Frank AL, Glick DH: The anticurvature lling method to pre- pare the curved root canal. J Am Dent Assoc, 1980; 101: 792-94.

03. Kandaswamy D, Venkateshbabu N, Porkodi I, Pradeep G: Canalcentering ability: An endodontic challenge. J Conserv Dent. Mumbai. 2009; 12: 3-9.

04. Peters OA: Current challenges and concepts in the preparation of root ca- nal systems: a review. J Endod. 2004; 30:559-67.

05. Iqbal MK, Maggiore F, Suh B et al: Comparison of apical transportation in four Ni-Ti rotary instrumentation technique. J Endod. 2003; 29: 587-91.

06. Glosson CR, Haller RH, Dove SB, Del Rio CE: A comparison of root canal preparations using NiTi hand, NiTi engine-driven, and K-Flex endodontic instruments. J Endod. 1995; 21: 146-51.

07. Pettiette MT, Delano EO, Trope M: Evaluation of success rate of endodon- tic treatment performed by students with stainless-steel K- les and nick- el-titanium hand les. J Endod. 2001; 27:124-27.

08. Gonzales-rodriguez MP, Ferrer-luque CM: A comparison of Pro le, Hero 642 and K3 instrumentation systems in teeth using digital imaging analysis. Oral Surg Oral Med Oral Pathol Oral Radiol Endod. 2004; 97:112-15.

09. Microgeorgis G, Molyvdas I, Lyroudia Ket al: A new methodology for the comparative study of the root canal instrumentation techniques based on digital radiographic image processing and analysis. Oral Surg Oral Med Oral Pathol Oral Radiol Endod. 2006; 101:125-31.

10. Hartmann MSM, Barletta FB, Camargo Fontanella VR, Vanni JR. Canal transportation after root canal instrumentation: a comparative study with computed tomography. J Endod; 2007; 33(8): 962-965.

11. Lofthag-Hansen S, Huumonen S, Gröndahl K, Gröndahl HG. Limited cone-beam CT and intraoral radiography for the diagnosis of periapical pathology. Oral Surg Oral Med Oral Pathol Oral Radiol Endod 2007; 103:114-9.

12. Cotton TP, Geisler TM, Holden DT, Schwartz SA, Schindler WG. Endodontic applications of cone-beam volumetric tomography. J Endod 2007; 33:1121-32.

13. Alves RA, Souza JB, Gonçalves Alencar AH, Pécora JD, Estrela C. Detection of proceduralerrors withstainlesssteel and NiTiinstruments by undergraduate students using conventional radiograph and cone beam computed tomography. Iran Endod J 2013; 8:160-5.

14. Estrela C, Bueno MR, Sousa-Neto MD, Pécora JD. Method for determination of root curvature radius using cone-beam computed tomography images. Braz Dent J 2008; 19:114-8. 
15. Mamede-Neto I, Borges AH, Guedes OA, Oliveira D, Pedro FLM, Estrela C. Root Canal Transportation and Centering Ability of Nickel-Titanium Rotary Instruments in Mandibular Premolars Assessed Using Cone- Beam Computed Tomography. The Open Dent J. 2017; 11:71-78.

16. Merrett SJ, Bryant ST, Dummer PM. Comparison of the shaping ability of RaCe and FlexMaster rotary nickel-titanium systems in simulated canals. J Endod. 2006; 32:960-2.

17. Hashem AA, Ghoneim AG, Lutfy RA, et al. Geometric analysis of root canals prepared by four rotary NiTi shaping systems. J Endod. 2012; 38: 996-1000.

18. Blum JY, Machtou P, Ruddle C, Micallef JP. Analysis of mechanical preparations in extracted teeth using ProTaper rotary instruments: value of the safety quotient. J Endod. 2003; 29: 567-75.

19. Yoshiminey, Ono M, Akamine A. The shaping effects of three nickeltitanium rotary instruments in simulated S-Shaped canals. J Endod. 2005; 31: 373-5.
20. Javaheri HH, Javaheri GH. A comparison of three Ni-Ti rotary instruments in apical transportation. J Endod. 2007; 33: 284 - 6 .

21.21 - Buchanan LS. The standardized-taper root canal preparationPart 6. GT le technique in abruptly curved canals. Int Endod J. 2001; 34(3):250-59.

22. Schäfer E, Vlassis M. Comparative investigation of two rotary nickeltitanium instruments: ProTaper versus RaCe. Part 1. Shaping ability in simulated curved canals. Int Endod J. 2004; 37: 229-38.

23. Rangel S, Cremonese R, Bryant S, Dummer P. Shaping ability of RaCe rotary nickel-titanium instruments in simulated root canals. J Endod. 2005; 31:460-3.

24. Hülsmann M, Peters O, Dummer PMH. Mechanical preparation of root canals: shaping goals, techniques and means. Endodontic Topics 2005; 10:30-76.

\section{ABSTRACT}

Aim: This study evaluated root canal transportation after root canal instrumentation with two nickel-titanium (NiTi) rotary systems, by using cone beam computed tomography (CBCT) imaging. Material and Methods: Mesiobuccal and mesiolingual root canals of twenty human mandibular molars were randomly divided into two experimental groups $(n=10)$, according to the NiTi rotary system: Group 1 - BioRace; and Group 2 - ProTaper Next. CBCT scans were obtained before the root canal instrumentation (CBCT 1) and after the use of BR2 (\#25/.04) and X2 (\#25/.06) instruments (CBCT 2) and after the use of BR5 (\#40/.04) and X4 (\#40/.06) instruments (CBCT 3). Two examiners evaluated the occurrence or not of transportation. Measurements were made at 3 different points: 1-2 $\mathrm{mm}$ from the apex (apical third);
3-4 $\mathrm{mm}$ from the apex (middle third); 4- $2 \mathrm{~mm}$ below furcation (cervical third). The Kruskal-Wallis and Dunn tests were used for statistical analysis. The level of significance was set at 5\%. Results: Root canals instrumented with BioRace instruments showed no transportation $(\mathrm{P}<0.05)$. Transportation was observed only in the apical third of the mesiobuccal root canal after the use of ProTaper Next X4 instrument $(\# 40 / .06)(\mathrm{P}<0.05)$. Conclusions: The BioRace system allowed the widening of mesiobuccal and mesiolingual root canals of mandibular molars up to apical diameter 40 without resulting in root canal transportation.

Keywords: Endodontics, Dental Instruments, Cone-Beam Computed Tomography.

\section{AUTOR PARA CORRESPONDÊNCIA}

Gustavo Silva Chaves

Faculdade de Odontologia, Universidade Federal de Goiás

Praça Universitária s/n, Setor Universitário

CEP 74605-220 Goiânia, Goiás.

Tel: (62) 3209-6254 